\title{
Dual Testing to Achieve Low On-treatment Platelet Reactivity for Aneurysm Embolization
}

\author{
Halitcan Batur ${ }^{1}$ Mehmet A. Topcuoglu' $\cdot$ Sinan Balci ${ }^{3} \cdot$ Ethem M. Arsava $^{2} \cdot$ Anil Arat $^{3}$ \\ Received: 20 December 2020 / Accepted: 8 March 2021 \\ (c) Springer-Verlag GmbH Germany, part of Springer Nature 2021
}

\begin{abstract}
Background and Purpose Although point-of-care tests are used extensively to test platelet function before endovascular aneurysm treatment, their use and validity are still debated. We compared the results of two point-of-care tests (VerifyNow ${ }^{\circledR}$ and Multiplate ${ }^{\circledR}$ ) for assessing patients treated with stents and flow diverters and determined their relation to periprocedural complications.

Methods All patients undergoing treatment of intracranial aneurysms were tested using both methods and were retrospectively evaluated. Patients with acute subarachnoid hemorrhage and those who had to be maintained on anticoagulants for unrelated diseases were excluded. An acceptable level of platelet inhibition was required on both tests to commence with treatment, otherwise antiplatelet medication was adjusted to reach this level.

Results Mean PRU (platelet reactivity units) and ADP AUC (adenosine diphosphate area under the aggregation curve) were $68 \pm 66$ and $23 \pm 15$, respectively, in 295 patients. Both tests showed a good correlation $(r=0.45)$. Both tests were able to predict hemorrhagic events but not ischemic events. When patients with very low reactivity $(P R U<60)$ were compared to the rest of the group, there were more hemorrhagic events in the first group but the overall rate of complications were similar $(p=0.27)$.

Conclusion In this largest study comparing two widely used commercial platelet function tests, the correlation between the tests were less than ideal; however, the very low platelet reactivity attained by the help of dual platelet testing did not result in an increased overall complication rate.
\end{abstract}

Keywords Hemorrhage $\cdot$ Intracranial aneurysm $\cdot$ Complication $\cdot$ PRU $\cdot$ ADP

\section{Introduction}

In the endovascular treatment of intracranial wide-necked or nonsaccular aneurysms, stents and flow diverters are frequently used, especially when coiling with or without balloon assistance would be a challenging procedure [1]. Due to the bare metal components of stents, intraoperative, pre-

Availability of Data and Material Data is available from the corresponding author upon reasonable request.

\footnotetext{
Anil Arat

anilarat@hotmail.com

1 Department of Radiology, Ministry of Health Ankara City Hospital, Ankara, Turkey

2 Department of Neurology, Hacettepe University Hospitals, Ankara, Turkey

3 Department of Radiology, Hacettepe University Hospitals, Ankara, Turkey
}

operative and postoperative antiaggregation is essential [2, $3]$. On the other hand, antiaggregation may result in hemorrhagic complications, including intracranial hemorrhage [4]. Acetylsalicylic acid (ASA) and clopidogrel dual therapy is the standard treatment method for antiaggregation when intra-arterial scaffolds are used. The response to the more potent of these medications, clopidogrel, is highly variable between individuals [5]. Therefore, platelet function tests are used both preoperatively and in postoperative follow-up to assess the on-treatment platelet reactivity [6]. There are contradictory opinions regarding the necessity and sufficiency of platelet function tests [7,8]. The aim of this retrospective study was to compare the relation of the results of two point-of-care tests (VerifyNow ${ }^{\circledR}$, ITC, Edison, NJ, USA; and Multiplate ${ }^{\circledR}$, Roche Diagnostics, Mannheim, Germany) for assessing patients undergoing intracranial aneurysm treatment with stents and flow diverters and determining their relation to periprocedural complications. 


\section{Material and Methods}

\section{Study Population}

Patients who had elective endovascular treatment of unruptured or remotely ruptured (more than 8 weeks after rupture) intracranial aneurysms using intracranial stents or flow-diverters were retrieved from the hospital information system. After a review of the data, patients taking only ASA and clopidogrel/prasugrel preoperatively were included; those who were already on anticoagulants or those who were started on anticoagulants after the procedure were excluded.

\section{Properties of Point-Of-Care Platelet Function Tests Used and Blood Sampling Protocol}

VerifyNow ${ }^{\circledR}$ is a point-of-care (POC) test that relies on the ability of activated platelets to bind to fibrinogen. This test has two parameters: BASE represents the specific aggregation to PAR1 and PAR4 thrombin receptors and corresponds to the state before starting antiaggregation therapy. Platelet reaction unit (PRU) values reflect the on-treatment platelet reactivity. By using adenosine diphosphate (ADP) as an agonist and PGE1 as a suppressor, the nonspecific effect of $\mathrm{P} 2 \mathrm{Y} 1$ is eliminated, thus rendering VerifyNow ${ }^{\circledR}$ more specific. Blood samples were collected in a $3.2 \%$ citrate tube.

Multiplate ${ }^{\circledR}$ also has two parameters, resembling the former test. Thrombin receptor activating peptide (TRAP) relates to the basal state (as in BASE) and ADP is the second parameter and indicates the on-treatment platelet reactivity. Parameters are quantified by area under aggregation curve (AUC) and arbitrary aggregation units (AU). The AUC is expressed in units (1 U corresponds with $10 \mathrm{AU}^{*} \min$ ) [9]. Inhibition of PGE1 is not used in the calculation of the last parameter, and blood is collected into a hirudinized tube.

After the patients had fasted overnight, venous blood was collected using a Vacutainer (Becton Dickinson, Franklin Lakes, NJ, USA) in the following order: a citrated tube for VerifyNow, a hirudinized tube for Multiplate, another citrated tube for activated partial thromboplastin time (aPTT) and prothrombin time (PT), and an EDTA-containing tube for complete blood count. Blood sampling was performed within $24 \mathrm{~h}$ before the treatment, and no additional antiplatelet therapy was administered after blood sampling.

\section{General Description of Endovascular Procedures}

Before the procedures, informed written consent was obtained from all patients. Patients were started on $75 \mathrm{mg}$ clopidogrel and $300 \mathrm{mg}$ ASA at least 5 days before the procedure. In cases of high on-treatment platelet reactivity (HPR), the procedure was postponed, antiaggregation therapy was changed to $10 \mathrm{mg} /$ day prasugrel and the same dose of aspirin for 5 more days, and measurements were repeated before the procedure. For these patients, only the second measurements were included in the study. The level of ontreatment platelet reactivity was assessed by VerifyNow ${ }^{\circledR}$ (ITC) and Multiplate ${ }^{\circledR}$ (Roche Diagnostics) from the same blood specimen. Using a coaxial system consisting of a $6 \mathrm{~F}$ introducer sheath and a distal access/intermediate catheter, stents and flow diverters were installed. In stent-assisted coiling cases, a second microcatheter was introduced into the aneurysm and was jailed by the stent. All patients were anticoagulated with IV heparin during the procedure, starting with a 70-100 IU/kg IV bolus dose just after insertion of the introducer sheath, followed by infusions to achieve an activated clotting time approximately twice that of the baseline. In cases of an impending or definite thromboembolic event during the procedure, patients were started on IV tirofiban. Hemostasis at the arterial puncture side was achieved by deployment of an AngioSeal VIP vascular closure device (Terumo Medical Corporation, Tokyo, Japan).

\section{Definition of HPR, LPR and VLPR}

We defined the platelet reactivity groups in our study based on the PRU levels as per the previous work of Delgado Almandoz et al. [6] in the following manner: PRU > 240: high on-treatment platelet reactivity (HPR), $240 \geq P R U \geq 60$ : low on-treatment platelet reactivity (LPR), PRU $<60$ : very low on-treatment platelet reactivity (VLPR).

Additionally, the cut-off value for area under aggregation curve (AUC) for ADP was set at 40 as previously described by Flechtenmacher et al. [10] (ADP AUC > 40: HPR, ADP AUC $\leq 40$ : LPR).

\section{Data Collection and Processing}

Preoperative and perioperative data, including 30-day outcomes, were collected. Clinical data (sex, age, body weight, history of smoking, malignancy, hyperlipidemia, hypertension, diabetes mellitus, thrombophilia) and laboratory data, e.g. PRU, BASE, TRAP, ADP, MPV (mean platelet volume), hematocrit and platelet count were collected. Intraoperative and perioperative hemorrhagic or thrombotic complications and the use of intraoperative tirofiban were also noted. The following were included as hemorrhagic complications: arterial puncture site hemorrhage causing hemodynamic instability or necessitating further medical intervention and any other extracranial hematoma and periprocedural subarachnoid or intraparenchymal hemorrhage. The following events were classified as periprocedural (30 days) ischemic complications: thrombus formation in the stent, parent artery or side branch thrombotic occlusion or distal emboli and acute myocardial infarction. In some patients treated with flow diversion, temporary self-limited flow-re- 
lated nonopacification of a jailed side branch (the so-called Coanda effect) occurred. Additionally, the side branch was intentionally sacrificed in some patients with communicating artery or carotid tip aneurysms by encroaching onto the side branch origin with coils, provided that there was previously documented good collateral flow via the communicating arteries. These latter two conditions were asymptomatic in all cases and were not included as thrombotic complications.

\section{Statistics}

All values are displayed as the mean \pm standard deviation and percentages. The Mann-Whitney $U$-test, Student's $t$ test, $\chi^{2}$-test and Fisher's exact tests, and ANOVA with post hoc Tukey's test were used as appropriate. Receiver operating characteristic (ROC) curves were generated to determine the concordance of two tests and determine their thresholds. Areas under the curve (AUC) and their 95\% confidence intervals $(95 \% \mathrm{CI})$ were reported. The correlation between two tests was determined with the concordance correlation test and Band-Altman graphical plotting. A $p$ value lower than 0.05 was set as the level of statistical significance. The SPSS ${ }^{\circledR} 22.0$ (IBM, Armonk, New York, USA) statistical package program was used for statistical analysis.

\section{Results}

\section{Study Population}

A total of 321 adult patients (mean age: $51 \pm 13$ years, body weight: $72 \pm 12 \mathrm{~kg}, 201$ female) were included. Of these, 295 had simultaneous preprocedural VerifyNow and Multiplate determination of on-treatment platelet reactivity within $24 \mathrm{~h}$ of the start of the procedure. All aneurysms were either unruptured or beyond the acute phase of subarachnoid hemorrhage. The mean PRU was $68 \pm 66$ (range: 0-240) and the mean ADP AUC was $23 \pm 15$ (range: $1-86$ ). Of the patients 130 had VLPR, 165 patients had LPR and none of the patients had HPR according to PRU and 37 patients had HPR according to ADP AUC. Of the patients 16 had the antiplatelet therapy changed to prasugrel (mean PRU: $4.5 \pm 9$ and mean ADP AUC: $15 \pm 9$ ). For the whole cohort, the mean platelet count was $242 \pm 6910^{3} / \mathrm{ml}$, the mean platelet volume (MPV) was $8.1 \pm 1.3$ and the mean hematocrit was $38.7 \pm 6.6$. In five patients, the Multiplate assay suggested marginally high platelet reactivity. Since these five patients had low platelet reactivity according to the VerifyNow assay, they were treated endovascularly on the same day at the discretion of the operator and subsequently included in this study.
At least 1 procedural adverse event was noted in 31 $(9.6 \%)$ patients. Hemorrhagic complications were seen in 8 $(2.5 \%)$ procedures, which resulted in 1 mortality. Ischemic complications were noted in $20(6.2 \%)$ patients, which resulted in 4 permanent morbidities (Supplementary table 1). Out of the 16 patients whose therapy was changed to prasugrel 1 had a hemorrhagic complication. Three events (significant exacerbation of aneurysm mass effect in two patients and parent artery occlusion due to postprocedural "watermelon seeding" of a flow diverter in one patient) could not be classified as either hemorrhagic or ischemic, as they did not have a direct relation to antiplatelet therapy. These events resulted in one mortality and one morbidity, and the parent artery occlusion was not symptomatic. The overall rate of mortality and permanent morbidity was $2.7 \%$. No difference was noted in terms of demographic variables, risk factors or hematological profiles in these complicated (either ischemic or hemorrhagic) procedures compared to uneventful procedures. Of the patients who were included in the ischemic complication group 13 were administered intraoperative tirofiban due to thrombus formation in the stent after proper placement. Further hemorrhagic adverse events were not noted in these 13 patients.

\section{Correlation Between Two Tests}

The PRU and ADP AUC measurements showed a good correlation (correlation coefficient, $r=0.45$, 95\% CI: 0.52-0.39, $p<0.0001$, Fig. 1a). The Bland-Altman plot (Fig. 1b) showed that the vast majority of values did not exceed the maximum allowed difference $( \pm 1.96 \mathrm{SD})$ between methods, indicating a reasonable level of agreement.

\section{Ischemic Complications}

Since a meticulous preprocedural adjustment of LPR or VLPR had been attained, ROC analysis could not identify the risk threshold of ischemic complications for both methods. The area under curve (AUC) was 0.57 (95\% confidence interval: $0.511-0.627, p=0.308$ vs. area $=0.5)$ for ADP AUC and 0.615 (95\% confidence interval: 0.553-0.675, $p=0.08$ vs. area $=0.5$ ) for PRU. PRU and ADP AUC values were not significantly different among patients with uneventful procedures (ADP AUC; $23 \pm 15$ vs. $25 \pm 17$, $p=0.323$ ) (PRU; $68 \pm 67$ vs. $84 \pm 55, p=0.09$ ) (Supplementary table 2).

\section{Hemorrhagic Complications}

Unlike ischemic complications ROC analysis could identify the risk threshold for hemorrhagic complications. The AUC was 0.692 (95\% confidence interval: $0.636-0.744, p=0.04$ vs. area $=0.5)$ for ADP AUC and $0.66(95 \%$ confidence in- 
a

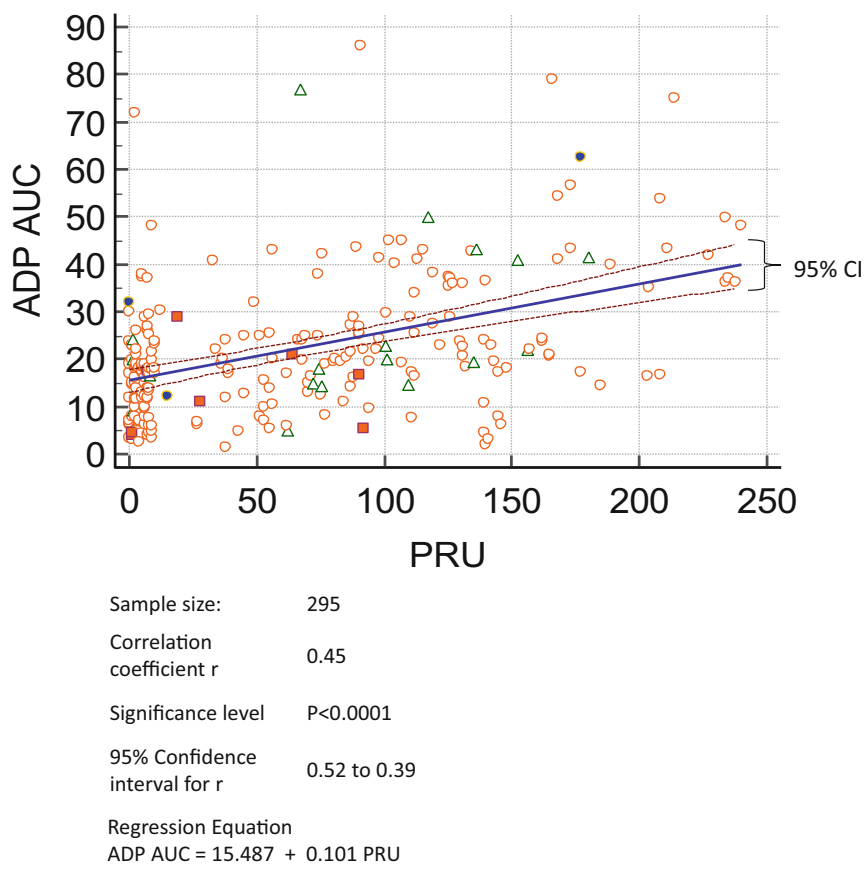

b

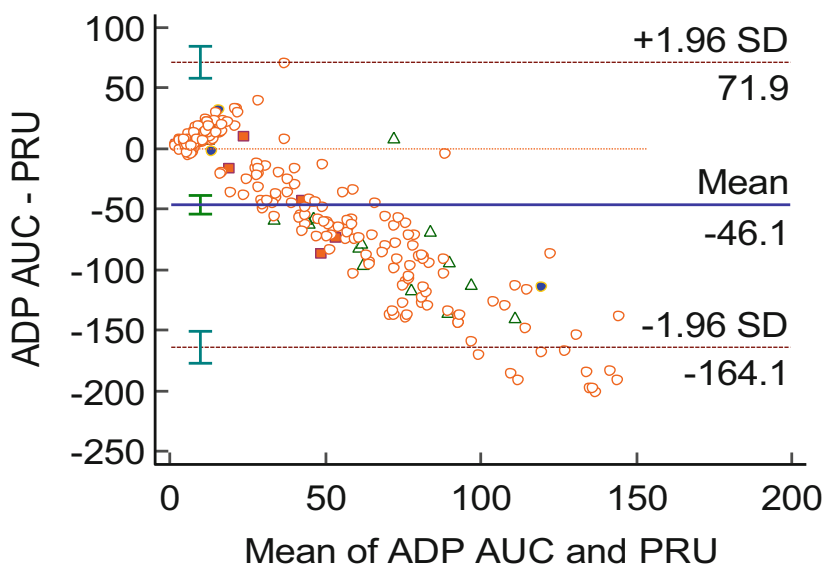

Fig. 1 a Scatter plot showing the concordance correlation between ADP AUC and PRU. X axis: PRU, Y axis ADP AUC. Patients with ischemic complications are denoted by green triangles, those with hemorrhagic complications are marked as red squares. Blue dots represent complications that cannot be classified as strictly ischemic or hemorrhagic. Red circles are patients without any complications. b Bland-Altman plot for agreement between PRU and ADP levels. Patients with ischemic complications are denoted by green triangles, those with hemorrhagic complications are marked as red squares. Blue dots represent complications that cannot be classified as strictly ischemic or hemorrhagic. Red circles are patients without any complications. ADP AUC adenosine diphosphate area under the aggregation curve, $P R U$ platelet reactivity units

terval: $0.61-0.71, p=0.035$ vs. area $=0.5$ ) for VerifyNow. Interestingly PRU was not able to identify a risk threshold for hemorrhagic complications (AUC $=0.63[95 \%$ confidence interval: $0.561-0.682], p=0.15$ vs. area $=0.5$ ).

The optimal threshold value for ADP AUC was 17, with a sensitivity of $75 \%$ (95\% confidence interval: 46.9-90.8\%) and specificity of 58\% (95\% confidence interval: 58.3-69.7\%). In addition, the sensitivity of ADP AUC reached $100 \%$ (95\% confidence interval: $64-100 \%$, specificity: $25.3 \% 95 \%$ confidence interval: $19.5-29.7 \%$ ) for a threshold of 29 , denoting that bleeding occurs under this value.

The optimum bleeding threshold for VerifyNow was found to be $63 \%$ with a sensitivity of $100 \%$ (95\% confidence interval: $64-100)$ and specificity of $36.2 \%$ (95\% confidence interval: 31-42); exceeding this cut-off would increase bleeding risk.

ADP AUC was significantly lower and percentage VerifyNow was significantly higher in patients with hemorrhagic complications when compared to those without complications (ADP AUC: $13 \pm 9$ vs. $23 \pm 15, p=0.027$ ) (VerifyNow: $86 \pm 14 \%$ vs $72 \pm 26 \%, p=0.026$ ). PRU was numerically lower in patients with hemorrhagic complications but not statistically significant ( $37 \pm 40$ vs. $68 \pm 67, p=0.06$ ) (Supplementary table 2).

\section{Complications in High Versus Low Versus Very Low Platelet Reactivity Groups}

Accepting ADP AUC as a classifier, there was no statistically significant difference between ischemic, hemorrhagic and total complication rates between LPR and HPR cohorts; however, when PRU was used as a classifier, the ischemic complication rate was significantly lower in the VLPR group as compared to the LPR group (3\% vs. 9.6\%, $p=0.004)$ and the hemorrhagic complication rate was numerically higher in the VLPR group (but not statistically significant, $3.8 \%$ vs. $1.8 \%, p=0.38$ ). The overall complication rate was not significantly different between the VLPR and LPR groups ( $8.4 \%$ vs. $12 \%, p=0.27$, Supplementary table 3).

\section{Discussion}

To our knowledge, this is the largest study exclusive to intracranial aneurysm treatment procedures. In contrast to the prior studies, all treatments were performed by the same operator, enabling a homogeneous operational technique and elimination of procedural variables that may affect hemorrhagic and occlusive complication rates. In addition, both antiplatelet function tests were performed simultaneously 
from the same blood specimen, eliminating the potential effects of blood withdrawal technique.

Our results show that the correlation between the widely adopted point-of-care tests is not ideal and that neither can predict the occurrence of thrombotic complications in a LPR patient population. This is not a surprising result since it has been demonstrated that VerifyNow results are not obstinate; our results suggest that this likely holds true for Multiplate as well. Inconsistencies in VerifyNow measurements were reported in a study by Bender et al. [11], who showed that $24 \%$ of patients had fluctuations in the VerifyNow results during repeated same day tests. The same group of authors also found that the procedural complexity of flow diversion rather than platelet reactivity as measured by point-of-care testing can predict thrombotic complications [12].

Therefore, dual testing using both VerifyNow and Multiplate may help in identifying the risk of hemorrhagic complications better than a single test. The VerifyNow and Multiplate tests can be used interchangeably in most patients to detect the antiplatelet activity of thienopyridines, such as clopidogrel and prasugrel; however, the correlation between the two tests is not ideal, and the results may differ in a certain proportion of patients. Unfortunately, the basic characteristics of the patients, such as age, gender, weight, atherosclerosis risk factor status and hematological basic parameters, were not helpful in predicting the variance between the two tests. At the first glance, these results may suggest that dual testing may be futile; however, in real life practice, when an interventionist is faced with an unacceptably low or high platelet reactivity prior to an endovascular procedure, dual testing enables proceeding with minimal alteration of the antiplatelet therapy, provided that at least one of the point of care tests indicates acceptable platelet inhibition. Thus, alteration of the APT or, alternatively, referral to perform a more complex test such as light transmission aggregometry (LTA) will be necessary only for those patients who have unacceptably low or high antiplatelet inhibition levels on both tests performed on the same blood specimen. This is also a convenient method for the patient as it saves time, effort, and costs and limits the number of blood withdrawals.

Another difference between our study and previous studies is that the on-treatment platelet reactivity is lower in our study compared to the findings of Goh et al. [4] (median $30 \%$, range $0-99 \%, 72 \%$ is accepted as clopidogrel hyperresponse). Kashiwazaki et al. [13] also suggested that the best results are obtained in patients with 26-74\% inhibition based on VerifyNow. In our study, the mean inhibition percentage assessed by VerifyNow was $72 \pm 24 \%$. Furthermore, the mean PRU value $(68 \pm 66)$ in our study mostly overlapped with those of the hyperresponder groups of the previous studies $[6,10,14,15]$. This is because we in- tentionally erred on the side of an LPR. The underlying reason for this preference is that hemorrhagic events are less frequently observed than thrombotic events in independently adjudicated trials with flow diverters. For example, in the pipeline for uncoilable or failed aneurysms (PUFS) [16] trial, which included clopidogrel and aspirin dual antiplatelet therapy without the use of a platelet function test, 13 of the 108 patients $(12 \%)$ suffered serious thrombotic adverse effects, such as carotid occlusion, ischemic stroke, and carotid occlusion, whereas only 5 patients (4\%) suffered from intracranial hemorrhage, 1 of which was actually posttraumatic. Similarly, the first of a series of randomized trials, the flow diversion in the treatment of intracranial aneurysm trial (FIAT) [17], reported a similar rate of thrombotic events versus hemorrhagic events in the flow diversion group. The second randomized study, the parent artery reconstruction for large or giant cerebral aneurysms using the Tubridge flow diverter (PARAT) trial [18], reported a total of $7(4.8 \%)$ hemorrhagic events versus 14 (9.7\%) ischemic complications. First year outcomes of the recent and independently adjudicated SCENT trial [19] showed a $10.6 \%$ rate of new or worsening stroke versus a $2.2 \%$ hemorrhagic risk.

Dual testing and consequent selection of patients or modification of antiplatelet therapy allowed us to achieve uniformly low platelet reactivity, which is one of the lowest reported in the literature if not the lowest. Hence, we were able to examine the results of a subset of patients with VLPR, similar to those patients who are primarily premedicated by new and more potent antiplatelet agents such as prasugrel or ticagrelor [20, 21]. This subset of patients are generally excluded in other studies as very low reactivity is considered by some to be a contraindication for endovascular treatment. When patients with VLPR were compared to the remaining patients, we found that treatment under VLPR was not necessarily linked to a higher overall complication rate, despite a somewhat higher rate of hemorrhagic complications. This suggests that modification of antiplatelet therapy may not be absolutely necessary when VLPR levels are encountered. Besides being less common than thrombotic complications, hemorrhagic complications may be caused by various technical factors, which are not necessarily in direct relevance with the level of platelet inhibition (e.g. wire perforation or hyperperfusion are not directly related to low platelet reactivity but these complications may result in worse prognosis in patients with highly inhibited platelets). In addition, with the advent and more liberal use of more delicate and softer yet more metallic devices, such as intravascular and intrasaccular flow diverters, it may be expected that the risk of hemorrhagic complications will decrease but thrombotic complications will increase over time. This potentially necessitates lower lev- 
els of on-treatment platelet reactivity, which can be attained via the use of dual antiplatelet testing.

Our study has several limitations. First, aspirin responsiveness was not evaluated. Second, other platelet function tests, such as LTA (the gold standard test for platelet function) [22] and thromboelastography [23], were not included in our study. Among these two tests, LTA was shown to better correlated with thromboembolic complications in cerebrovascular procedures in a critical study by Flecthenmacher et al., who mainly evaluated patients with carotid bifurcation/intracranial stenting (93 patients) rather than intracranial aneurysm embolization (only 10 patients as opposed to 295 patients in our study) [10]; however, these two tests are more complex, requiring lengthy and semiautomated processing in comparison to fully automated, userfriendly point-of-care tests included in our study [24]. Finally, the ischemic and hemorrhagic complication rates in our study and in the current literature are not high. Due to the limited number of index events, a large population size is needed to avoid neglecting differences related to on-treatment platelet reactivity within the limited number of events. For this reason and for practical purposes, large-scale multicenter registries about the effects of platelet reactivity testing on the results of endovascular aneurysm treatment need to be started.

\section{Conclusion}

There is no consensus on the level of on-treatment platelet reactivity as determined by point of care assays in interventional neuroradiology practice. VerifyNow and Multiplate, two widely used point-of-care assays, show a merely reasonable correlation; however, when these tests are used simultaneously, a state of very low platelet reactivity, similar to the state obtained by the newer antiplatelet agents (e.g. prasugrel and ticagrelor) can be attained. Endovascular aneurysm treatment under such lower on-treatment platelet reactivity appears to be feasible, yet a definite risk of bleeding should be acknowledged.

Supplementary Information The online version of this article (https:// doi.org/10.1007/s00062-021-01011-7) contains supplementary material, which is available to authorized users.

Author Contribution H. Batur and M. A. Topcuoglu made substantial contribution to acquisition, analysis and interpretation of data; E. M. Arsava and A. Arat took part in conception and design of study; A. Arat performed final editing of the manuscript; all authors approved the final version of the manuscript.

\section{Declarations}

Conflict of interest H. Batur, M. A. Topcuoglu, S. Balci, E. M. Arsava and A. Arat declare that they have no conflict of interests.
Ethical standards All procedures performed in this study involving human participants were in accordance with the ethical standards and were approved by the local ethics committee and comply with the 1975 Helsinki declaration and its later amendments or comparable ethical standards. Informed consent was obtained from all individual participants included in the study.

\section{References}

1. Galal A, Bahrassa F, Dalfino JC, Boulos AS. Stent-assisted treatment of unruptured and ruptured intracranial aneurysms: clinical and angiographic outcome. Br J Neurosurg. 2013;27:607-16.

2. Lee DH, Arat A, Morsi H, Shaltoni H, Harris JR, Mawad ME. Dual antiplatelet therapy monitoring for neurointerventional procedures using a point-of-care platelet function test: a single-center experience. AJNR Am J Neuroradiol. 2008;29:1389-94.

3. Drazin D, Choulakian A, Nuño M, Kornbluth P, Alexander MJ. Body weight: a risk factor for subtherapeutic antithrombotic therapy in neurovascular stenting. J Neurointerv Surg. 2011 Jun;3(2):177-81. https://doi.org/10.1136/jnis.2010.004085.

4. Goh C, Churilov L, Mitchell P, Dowling R, Yan B. Clopidogrel hyper-response and bleeding risk in neurointerventional procedures. AJNR Am J Neuroradiol. 2013;34:721-6.

5. Karaźniewicz-Łada M, Danielak D, Burchardt P, Kruszyna L, Komosa A, Lesiak M, Główka F. Clinical pharmacokinetics of clopidogrel and its metabolites in patients with cardiovascular diseases. Clin Pharmacokinet. 2014;53:155-64.

6. Delgado Almandoz JE, Crandall BM, Scholz JM, Fease JL, Anderson RE, Kadkhodayan Y, Tubman DE. Pre-procedure P2Y12 reaction units value predicts perioperative thromboembolic and hemorrhagic complications in patients with cerebral aneurysms treated with the Pipeline Embolization Device. J Neurointerv Surg. 2013;5 Suppl 3:iii3-10.

7. Comin J, Kallmes DF. Platelet-function testing in patients undergoing neurovascular procedures: caught between a rock and a hard place. AJNR Am J Neuroradiol. 2013;34:730-4.

8. Skukalek SL, Winkler AM, Kang J, Dion JE, Cawley CM, Webb A, Dannenbaum MJ, Schuette AJ, Asbury B, Tong FC. Effect of antiplatelet therapy and platelet function testing on hemorrhagic and thrombotic complications in patients with cerebral aneurysms treated with the pipeline embolization device: a review and metaanalysis. J Neurointerv Surg. 2016;8:58-65.

9. Müller-Schunk S, Linn J, Peters N, Spannagl M, Deisenberg M, Brückmann H, Mayer TE. Monitoring of clopidogrel-related platelet inhibition: correlation of nonresponse with clinical outcome in supra-aortic stenting. AJNR Am J Neuroradiol. 2008;29:786-91.

10. Flechtenmacher N, Kämmerer F, Dittmer R, Budde U, Michels P, Röther J, Eckert B. Clopidogrel Resistance in Neurovascular Stenting: Correlations between Light Transmission Aggregometry, VerifyNow, and the Multiplate. AJNR Am J Neuroradiol. 2015;36:1953-8.

11. Bender MT, Zarrin DA, Campos JK, Jiang B, Chandra A, Vo CD, Caplan JM, Huang J, Tamargo RJ, Lin LM, Colby GP, Coon AL. Precision of VerifyNow P2Y12 Assessment of Clopidogrel Response in Patients Undergoing Cerebral Aneurysm Flow Diversion. Neurosurgery. 2019;85:543-9.

12. Jiang B, Bender MT, Westbroek EM, Campos JK, Lin LM, Xu R, Tamargo RJ, Huang J, Colby GP, Coon AL. Procedural complexity independent of $\mathrm{P} 2 \mathrm{Y} 12$ reaction unit (PRU) values is associated with acute in situ thrombosis in Pipeline flow diversion of cerebral aneurysms. Stroke Vasc Neurol. 2018;3:169-75.

13. Kashiwazaki D, Kuwayama N, Akioka N, Hayakawa Y, Kuroda S. The roles and issues of P2Y12 percent inhibition assessed by VerifyNow assay for patients undergoing Neurointervention: a prospective study. J Stroke Cerebrovasc Dis. 2014;23:1830-6. 
14. Nishi $\mathrm{H}$, Nakahara I, Matsumoto $\mathrm{S}$, Hashimoto T, Ohta T, Sadamasa N, Ishibashi R, Gomi M, Saka M, Miyata H, Watanabe S, Okata T, Sonoda K, Kouge J, Ishii A, Nagata I, Kira J. Platelet reactivity and hemorrhage risk in neurointerventional procedures under dual antiplatelet therapy. J Neurointerv Surg. 2016;8:949-53.

15. Nakagawa I, Park HS, Yokoyama S, Wada T, Hironaka Y, Motoyama Y, Takayama K, Kichikawa K, Nakase H. Influence of Diabetes Mellitus and Cigarette Smoking on Variability of the Clopidogrel-Induced Antiplatelet Effect and Efficacy of Active Management of the Target P2Y12 Reaction Unit Range in Patients Undergoing Neurointerventional Procedures. J Stroke Cerebrovasc Dis. 2016;25:163-71.

16. Becske T, Brinjikji W, Potts MB, Kallmes DF, Shapiro M, Moran CJ, Levy EI, McDougall CG, Szikora I, Lanzino G, Woo HH, Lopes DK, Siddiqui AH, Albuquerque FC, Fiorella DJ, Saatci I, Cekirge SH, Berez AL, Cher DJ, Berentei Z, Marosfoi M, Nelson PK. LongTerm Clinical and Angiographic Outcomes Following Pipeline Embolization Device Treatment of Complex Internal Carotid Artery Aneurysms: Five-Year Results of the Pipeline for Uncoilable or Failed Aneurysms Trial. Neurosurgery. 2017;80:40-8.

17. Raymond J, Gentric JC, Darsaut TE, Iancu D, Chagnon M, Weill A, Roy D. Flow diversion in the treatment of aneurysms: a randomized care trial and registry. J Neurosurg. 2017;127:454-62.

18. Liu JM, Zhou Y, Li Y, Li T, Leng B, Zhang P, Liang G, Huang Q, Yang PF, Shi H, Zhang J, Wan J, He W, Liang C, Zhu G, Xu Y, Hong B, Yang X, Bai W, Tian Y, Zhang H, Li Z, Li Q, Zhao R,
Fang Y, Zhao K; PARAT investigators. Parent Artery Reconstruction for Large or Giant Cerebral Aneurysms Using the Tubridge Flow Diverter: A Multicenter, Randomized, Controlled Clinical Trial (PARAT). AJNR Am J Neuroradiol. 2018;39:807-16.

19. Meyers PM, Coon AL, Kan PT, Wakhloo AK, Hanel RA. SCENT Trial. Stroke. 2019;50:1473-9.

20. DeGrote JR, Olafson EM, Drofa A, Kouznetzov E, Manchak M, Leedahl ND, Leedahl DD. Ticagrelor and Acetylsalicylic Acid after Placement of Pipeline Embolization Device for Cerebral Aneurysm: A Case Series. Can J Hosp Pharm. 2018;71:349-55.

21. Choi HH, Lee JJ, Cho YD, Han MH, Cho WS, Kim JE, An SJ, Mun JH, Yoo DH, Kang HS. Antiplatelet Premedication for StentAssisted Coil Embolization of Intracranial Aneurysms: Low-Dose Prasugrel vs Clopidogrel. Neurosurgery. 2018;83:981-8.

22. Hvas AM, Favaloro EJ. Platelet Function Analyzed by Light Transmission Aggregometry. Methods Mol Biol. 2017;1646:321-31.

23. McTaggart RA, Choudhri OA, Marcellus ML, Brennan T, Steinberg GK, Dodd RL, Do HM, Marks MP. Use of thromboelastography to tailor dual-antiplatelet therapy in patients undergoing treatment of intracranial aneurysms with the Pipeline embolization device. J Neurointerv Surg. 2015;7:425-30.

24. Le Quellec S, Bordet JC, Negrier C, Dargaud Y. Comparison of current platelet functional tests for the assessment of aspirin and clopidogrel response. A review of the literature. Thromb Haemost. 2016;116:638-50. 